\title{
THE EFFECT OF DISTRACTORS ON STARTING-DELAY, MISSED OPPORTUNITIES AND UNSAFE CROSSINGS OF CHILDREN AND ADULTS IN A SIMULATED PEDESTRIAN CROSSING TASK
}

\author{
Zahra Tabibi \\ Assistant Professor in \\ Psychology \\ Department of Psychology \\ Ferdowsi University of Mashhad \\ Email: Iran tabibi@um.ac.ir
}

\author{
Karen Pfeffer \\ Senior Lecturer in Psychology \\ School of Psychology \\ University of Lincoln \\ United Kingdom \\ Email: kpfeffer@lincol.ac.uk
}

\begin{abstract}
:
The aims of the research were to investigate the effect of distractors on the pedestrian skills of children and adults. The pedestrian skill of deciding when it is safe to cross a road (decision-making skill) was assessed by a computer-presented simulated pedestrian task. It was predicted that distractors would reduce the performance of all age groups, with the reduction being greater for younger children. The pedestrian skills of Grade 2, Grade 4 and Grade 6 children and adults were assessed in three conditions, without distractors, with on-screen distractors and with off-screen distractors. The results showed that off-road distractors, whether visual or auditory, increased the starting-delay of all age groups. There was no significant difference between off-road-visual and off-road-auditory distractors for any age group except for Grade 6 children. Off-road-auditory distractors increased starting-delay more than off-road-visual distractors for Grade 6 children. Furthermore, off-road and on-road distractors increased the number of missed opportunities for Grade 2 children. On-road distractors increased the number of missed opportunities of Grade 4 and Grade 6 children. The increase was greater for Grade 6 children. The number of unsafe crossings was higher in the condition without distractors. Overall, decision-making skills were vulnerable to distractors. The degree of vulnerability differed depending on age and the specific measures of pedestrian skill.
\end{abstract}

KEYWORDS: Deciding when to cross, Visual distractors, Auditory distractors, Pedestrian injuries

\section{INTRODUCTION}

\subsection{Child pedestrian accidents}

Children and boys have disproportionately higher road accident rates (Roberts, Smith and Bryce,1995; Backett and Johnston, 1997; Peden et al., 2008). The type of road accidents that affect children is different to those affecting adults. While adults suffer mostly from car accidents, children have a higher rate of pedestrian accidents (Peden et al., 2008; Thomson, 1996a). There are three suggested means of reducing child pedestrian accidents, namely, Engineering, Enforcement, and Education (DETR, 1999).

Engineering techniques can be used to increase the safety of roads for users. Examples are the installation of pedestrian crossings, traffic calming measures, or pedestrian-traffic segregation (DETR,

1999). Engineers can assess road conditions, environmental factors and vehicle design features that could contribute to accidents (Thomson, 1991). Law enforcement is another way to increase safety; For example the 
enforcement of the use of roads instead of pavements for motorcycling (DETR,1999).

Some educational messages and programmes have been developed for drivers, parents and children in order to create safe traffic environments (DETR, 1999). However, their effectiveness has been challenged for not teaching what children really need to know in order to deal safely with traffic (AmpofoBoateng, Thomson, Grieve, Pitcairn, Lee and Demetre, 1993; Duperrex, Bunn and Roberts, 2002). The identification of the specific skills that children need to acquire in order to cope as pedestrians in traffic environments is urged.

\subsection{Pedestrian skill of deciding when to cross}

One specific skill for pedestrians is to decide when it is safe to cross. Whether it is safe or unsafe to cross is a decision that should be made on the basis of the time to contact judgment (the gap between the oncoming car and the pedestrian). The skills related to when to cross a road includes stopping at the kerb, looking at traffic, judging the traffic speed, delaying and deciding to cross. In this respect, the pedestrian should not be impulsive, too fast or too slow, at decision making (Plumert, Kearney \& Cremer, 2007; Seward, Ashmead \& Bodenheimer, 2007).

Zeedyk, Wallace and Spry (2002) found that $60 \%$ of their sample did not stop before proceeding on to the road. They suggested that distractibility is related to the behaviour of dashing out on to the street. Van der Molen (1981) reported that fifty percent of pedestrian accidents up to age 10 years were due to distraction (e.g., by an ice cream van). Interviewing children after an accident showed that $62 \%$ of boys and $50 \%$ of girls had an accident because of a complete lack of attention, and $41 \%$ of girls and

$26 \%$ of boys because of a partial lack of attention. Almost all children who had looked had also stopped (Van der Molen, 1981).
Dunbar, Lewis and Hill's (1999) research systematically showed how a lack of attentional skills could play a role in the observed risky behaviour of children when interacting with traffic situations. In addition, there are other studies that have shown children to be more conservative and overcautious than adults (Sheehy and Chapman, 1986). Foot et al. (1999) found that an increase of irrelevant information oriented the attention of both children and adults towards irrelevant information.

\subsection{Aims of the study}

Since in a natural traffic situation, different kinds of irrelevant information come from a variety of locations, the current study aimed to examine the effect of different types of distractors from different locations on the pedestrian skills of deciding when to cross (Decision-making skill). Decision-making skills were measured by length of startingdelay, number of missed opportunities, and number of unsafe crossings. The prediction was that distractors will increase the length of starting delay, number of missed opportunities and number of unsafe crossings. Distractors will have a more detrimental effect on the youngest age group than older children and adults. Those distractors located in places away from the main task would have a more detrimental effect than those located close to the main task.

\section{METHODS}

\subsection{Participants}

Eighty-eight children from three primary school grades, Grade 2, Grade 4 and Grade 6 participated in the study. Grade 2 children aged 6-7 years (29 children including 14 males and 15 females), Grade 4 children aged 8-9 years (30 children including 14 males and 16 females), and Grade 6 children aged 
10-11 years (29 children including 15 males and 14 females). Twenty-nine university students, including 10 males and 19 females, volunteered to participate in this study.

\subsection{Instruments}

A computer task was designed using measures based on previous studies of children's road safety skills (e.g., Lee, et al., 1984; AmpofoBoateng \& Thomson, 1991). It presented animated street scenes and was designed to be interactive and user-friendly for children. The experimental program was written using Microsoft Visual Basic 5 language, and designed to be displayed on a 1024 x 768 pixel resolution screen.

An image of a straight road was represented horizontally on the computer screen with the image of a boy standing at the kerb in the centre of the screen. Images of red cars of a constant size moved along the road (across the screen from right to left) at a constant speed with constant traffic sounds. The aim of the task was for the participants to move the figure across the road, as if they themselves are crossing the road. The boy moved across the road at a constant speed that could not be varied by the participant (e.g., the boy could not be made to "run" across the road).
Participants could move the boy across the road by pressing and holding down a button on a response key box. The next trial appeared automatically after the participant had made a response. There were twenty-one trials in total comprising three practice trials and eighteen test trials. The interval between each trial was two seconds.

The decision making task had three conditions differ in complexity: without distractors, with on-road distractors, and with off-road distractors. In this level, there were two different types of distractors. Visual distractors were located off the road but on the screen. Auditory distractors were located off the screen. There were two sets of this condition. Each set comprised of 10 trials including one practice trial. The sets were different in the order of presentation of roads and the combination of different distractors with the roads. The following tables show the distractors assigned to each trial for both sets. Set One included 4 trials with distractors located on the screen and 5 trials with distractors located off the screen. Set Two included 5 trials with distractors located on the screen and 4 trials with distractors off the screen.

Table 1: The order of images presented and the combination of different distractors with images for Set 1 .

\begin{tabular}{llll}
\hline Set 1 & & & \\
\cline { 1 - 3 } Trial & Distractors & Position of distractors & Type of distractors \\
\hline $\mathrm{p}$ & A gas balloon rising & On screen & Visual \\
1 & $\begin{array}{l}\text { A cartoon character jumping out from a } \\
\text { hole }\end{array}$ & On screen & Visual \\
& A sound of seabirds & Off screen & Auditory \\
2 & Cats fighting & Off screen & Auditory \\
3 & A cartoon character jumping out from a & On screen & Visual \\
4 & hole & & \\
& A boy shouting 'Again' & Off screen & Auditory \\
5 & Christmas tree with lights flashing & On screen & Visual \\
6 & A sound of a train & Off screen & Auditory \\
7 & A sound of marching & Off screen & Auditory \\
8 & Christmas tree with lights flashing & On screen & Visual \\
9 & & & \\
\hline
\end{tabular}




\section{RESULTS}

\subsection{Starting-delay}

Table 2 presents the data for starting-delay by age group, gender and conditions. As can be seen from the table, starting-delay decreased from Grade 2 to 4 then increased for Grade 6, with a decrease for the adult group. Therefore, no consistent decrement with age could be seen. Furthermore, an increase in starting-delay in conditions with-distractors can be seen. A GLM Repeated measures ANOVA was computed to see if these differences were significant.

Overall a Significant age-group effect emerged $(\mathrm{F}=9.7$, df $=3,105, \mathrm{p}<.001)$. Post hoc analyses using Tukey HSD indicated that the starting-delay of Grade 2,4 , and 6 children was significantly different from that of adults $(\mathrm{p}=.001, \mathrm{p}=$ $.02, \mathrm{p}=.001$ respectively). There were no significant differences in starting-delay between Grade 2, 4 and 6 children.
Overall a significant gender effect was observed $(\mathrm{F}=9.3$, df $=1,105, \mathrm{p}<.001)$, with males having a shorter starting-delay. Since there was no interaction with age group, all males of all age groups had a shorter starting-delay.

A significant condition effect emerged $(\mathrm{F}=$ 8.6, $\mathrm{df}=3,312, \mathrm{p}<.001)$. Pairwise comparisons using Bonferroni showed that, overall, starting-delay in withoutdistractors condition was not significantly different from conditions with on-road distractors, but it was shorter from both conditions with off- road-visual distractors $(\mathrm{p}=.004)$ and with off-road-auditory distactors $(\mathrm{p}=.001)$. Starting-delay in conditions with on-road distractors was significantly shorter than starting-delay in conditions with off- road-auditory distractors. In contrast with the prediction starting-delay in conditions with off-road visual and auditory distractors was not significantly different from each other $(\mathrm{p}=$ $.4)$.

Table 2: Mean and SD (in millisecond) of starting-delay of Grade 2, 4, 6 children separately for males and females by conditions.

\begin{tabular}{|c|c|c|c|c|c|c|c|c|c|}
\hline \multirow{3}{*}{$\begin{array}{l}\text { Age } \\
\text { group }\end{array}$} & \multirow{3}{*}{ Gender } & \multicolumn{8}{|c|}{ Conditions } \\
\hline & & \multicolumn{2}{|c|}{ Without-D } & \multicolumn{2}{|c|}{ With on-road } & \multicolumn{2}{|c|}{$\begin{array}{l}\text { With off-road- } \\
\text { visual D }\end{array}$} & \multicolumn{2}{|c|}{$\begin{array}{l}\text { With off-road- } \\
\text { auditory D }\end{array}$} \\
\hline & & M & SD & M & SD & M & SD & M & SD \\
\hline Grade & $\mathrm{M}$ & 1305.3 & 346.3 & 1408.8 & 593.0 & 1436.2 & 622.1 & 1676.8 & 942.0 \\
\hline \multirow[t]{2}{*}{2} & F & 1349.9 & 471.8 & 1397.6 & 409.5 & 1774.7 & 607.2 & 1758.6 & 592.7 \\
\hline & All & 1327.6 & 406.7 & 1403.2 & 500.1 & 1605.4 & 627.3 & 1717.7 & 773.4 \\
\hline Grade & M & 1189.5 & 348.7 & 1191.8 & 455.4 & 1272.1 & 320.4 & 1300.4 & 263.6 \\
\hline \multirow[t]{2}{*}{4} & $\mathrm{~F}$ & 1384.0 & 538.5 & 1282.9 & 419.9 & 1570.0 & 660.5 & 1646.4 & 563.1 \\
\hline & All & 1293.2 & 462.9 & 1240.4 & 431.6 & 1431.0 & 542.7 & 1484.9 & 475.4 \\
\hline Grade & $\mathrm{M}$ & 1236.7 & 542.4 & 1378.0 & 587.9 & 1418.8 & 608.3 & 1520.0 & 698.1 \\
\hline \multirow[t]{2}{*}{6} & $\mathrm{~F}$ & 1714.8 & 358.7 & 1560.9 & 577.8 & 1572.7 & 431.2 & 1790.5 & 483.7 \\
\hline & All & 1484.6 & 509.3 & 1472.9 & 578.9 & 1498.6 & 519.5 & 1660.2 & 600.8 \\
\hline \multirow[t]{3}{*}{ Adult } & M & 722.2 & 216.5 & 897.4 & 671.7 & 790.3 & 379.9 & 868.8 & 313.5 \\
\hline & $\mathrm{F}$ & 1027.2 & 320.5 & 1135.1 & 540.5 & 1238.4 & 475.3 & 1203.3 & 397.2 \\
\hline & All & 929.2 & 321.6 & 1058.7 & 584.1 & 1094.4 & 488.7 & 1095.8 & 399.5 \\
\hline
\end{tabular}




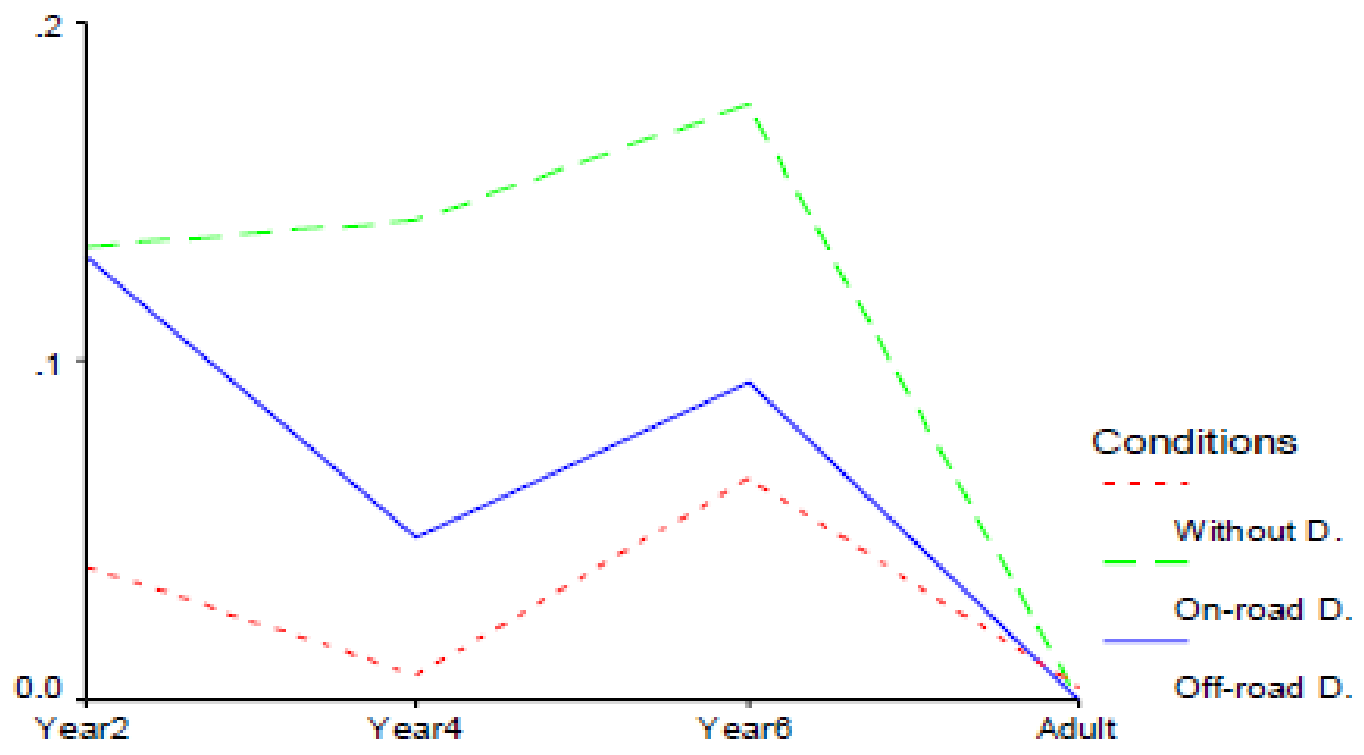

Age groups

D. = distractors

Figure 1: Starting-delay (millisecond) for each age group by conditions

\subsection{Missed opportunity}

Table 3 presents the average number of missed opportunities by age group under each condition.

The table shows that number of missed opportunities decreased with age up to Grade 4. There was an increase and a decrease again for Grade 6 and adults respectively. Therefore, no consistent age-related pattern is observable. In addition, the number of missed opportunities was higher in conditions with distractors. Nonparametric tests were used to assess differences by age group, gender and conditions.

For age group effect, the Kruskal-Wallis Test was used to examine age group differences on number of missed opportunities in all conditions combined. The analysis showed a significant difference $(\chi 2=$

15.827 , df $=3, p=.001$ ). Using Mann-Whitney $\mathrm{U}$ Test showed a significant difference between Grade 2 and adults $(\mathrm{z}=-3.8, \mathrm{p}=.001)$, Grade 4 and adults $(\mathrm{z}=-2.7, \mathrm{p}=.006)$, Grade 6 and adults $(\mathrm{z}=-3.6, \mathrm{p}$

$=.001)$, no significant difference between Grade 2 and Grade $6(\mathrm{z}=-.1, \mathrm{p}=.8)$, no significant difference between Grade 2 and Grade $4(\mathrm{z}=-1.2, \mathrm{p}=.2)$, and no significant difference between

Grade 4 and Grade $6(\mathrm{z}=-1.03, \mathrm{p}=.3)$.

For gender effect, a comparison between male and female subjects, overall, in number of missed opportunities was conducted using the Mann-Whitney U Test. The result of the analysis suggested that there was no significant difference between males and females in any condition: for conditions without-distractors $(\mathrm{z}=-1.691, \mathrm{p}=.09)$ for conditions with on-road distractors $(z=-1.669$, $\mathrm{p}=.095)$ or for conditions with off-road distractors $(\mathrm{z}=-1.288, \mathrm{p}=.2)$.

For conditions effect, the Friedman test was used to assess whether the increase in number 
of missed opportunities in conditions with distractors was significant. The analysis showed that, consistent with the first study, overall, the number of missed opportunities increased in conditions with on-road distractors $(\chi 2=15.88, \mathrm{p}=.001)$. This was not expected, as it was predicted that the number of missed opportunities would increase in conditions with off-road distractors because stronger distractors were included in this condition.

For interaction effect: As Figure 5-4 shows, the effect of different types of distractors on missed opportunities was different for different age groups. For that reason, a series of comparisons between conditions, without-distractors, with on-road distractors, with off-road distractors, separately for each age group was conducted using the Wilcoxon Signed Ranks Test. The result showed that:

For Grade 2 children, there was a significant difference in number of missed opportunities between conditions without-distractors and conditions with on-road distractors $(z=-2.079$, $\mathrm{p}=.04)$, and between conditions withoutdistractors and with off-road distractors $(\mathrm{z}=$ 2.023, $\mathrm{p}=.04)$. There was no significant difference between off-road and on-road conditions $(\mathrm{z}=-.472, \mathrm{p}=.6)$.

For Grade 4 children, there was a significant difference in number of missed opportunities between conditions without-distractors and conditions with on-road distractors $(z=-2.375$, $\mathrm{p}=.02)$. There was no significant difference between conditions without-distractors and with off-road distractors $(\mathrm{z}=-1.706, \mathrm{p}=.09)$. There was no significant difference between off-road and on-road conditions $(\mathrm{z}=$ -

$1.482, \mathrm{p}=.1)$.

For Grade 6 children, there was a significant difference in number of missed opportunities between conditions without-distractors and conditions with on-road distractors $(z=-2.689$, $\mathrm{p}=.007)$. There was no significant difference between conditions without-distractors and with off-road distractors $(\mathrm{z}=-1.886, \mathrm{p}=.06)$.
There was a significant difference between offroad and on-road conditions $(\mathrm{z}=-2.280, \mathrm{p}=$ $.02)$.

These analyses suggest that the number of missed opportunities for Grade 2 children increased in both conditions with distractors. However, the number of missed opportunities for Grade 4 and 6 children increased more in conditions with on-road distractors. The increase in number of missed opportunities in conditions with on-road distractors was greater for Grade 6 children. That is because there was a significant difference between on-road and off-road conditions for Grade 6 children, but not for Grade 4 children. Even though stronger distractors were included in this study, the result was compatible with the second study.

\subsection{Unsafe Crossing}

Since the number of occurrences of unsafe crossings was low, a comparison between conditions was not possible. Thus, no assessment of an interaction effect could be undertaken. However, Table 4 presents the number of participants in each age group that had at least one unsafe crossing in each condition. As can be seen from the table, the number of cases having at least one unsafe crossing decreased with age in all conditions. Consistent with the first and second studies, the number of cases having unsafe crossing was higher in conditions without-distractors.

Table 4: Number of participants having at least one unsafe crossing by condition

\begin{tabular}{|c|c|c|c|c|}
\hline & Grade & Grade & Grade & Adults \\
\hline Without D & 16 & 12 & 6 & 3 \\
\hline With on-road D & 4 & 1 & 0 & 1 \\
\hline With off-road D & 7 & 5 & 4 & 3 \\
\hline $\begin{array}{l}\text { off-road- } \\
\text { visual }\end{array}$ & 6 & 3 & 4 & 2 \\
\hline $\begin{array}{l}\text { off-road- } \\
\text { auditory }\end{array}$ & 4 & 3 & 2 & 2 \\
\hline All cases & 19 & 13 & 6 & 5 \\
\hline
\end{tabular}


Table 3: Mean (SD) number of missed opportunities separately for each age group under each condition

\begin{tabular}{|c|c|c|c|c|c|c|c|c|c|c|}
\hline \multirow[b]{2}{*}{ Conditions } & \multicolumn{2}{|c|}{ Grade 2} & \multicolumn{2}{|c|}{ Grade 4} & \multicolumn{2}{|c|}{ Grade 6} & \multicolumn{2}{|l|}{ Adult } & \multicolumn{2}{|c|}{ All cases } \\
\hline & Mean & $\begin{array}{l}S \\
D\end{array}$ & Mean & $\begin{array}{l}S \\
D\end{array}$ & Mean & $\begin{array}{l}\mathrm{S} \\
\mathrm{D}\end{array}$ & Mean & $\begin{array}{l}S \\
D\end{array}$ & Mean & S D \\
\hline Without D & .04 & .11 & .01 & .03 & .07 & .19 & .01 & .02 & .03 & .11 \\
\hline On-road D & .13 & .29 & .14 & .34 & .18 & .33 & .00 & .00 & .11 & .28 \\
\hline Off-road D & .13 & .27 & .05 & .15 & .09 & .19 & .00 & .00 & .07 & .19 \\
\hline off-visual D & .16 & .33 & .07 & .19 & .11 & .19 & .00 & .00 & .08 & .22 \\
\hline off-auditory $D$ & .11 & .25 & .03 & .14 & .11 & .23 & .00 & .00 & .06 & .19 \\
\hline All conditions & .30 & .60 & .20 & .43 & .34 & .68 & .01 & .02 & .21 & .50 \\
\hline
\end{tabular}

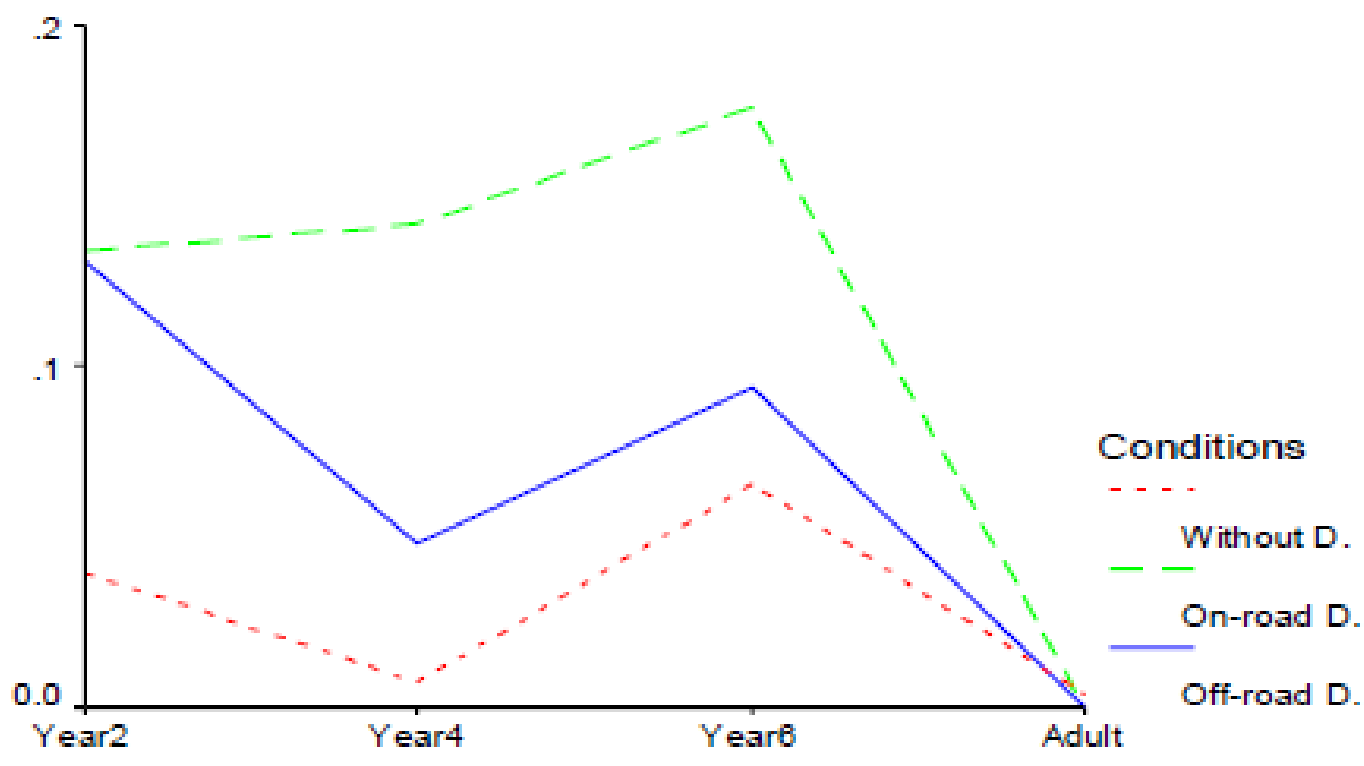

Age groups

D. = distractors

Figure 2: Number of missed opportunities by conditions for each age group 


\section{DISCUSSION}

The current study aimed to examine the effect of different types of distractors from different locations on the pedestrian skills of deciding when to cross (Decision-making skill). Decision-making skills were measured by length of starting-delay, number of missed opportunities, and number of unsafe crossings. The following results were obtained. With regard to starting-delay, off-road distractors, whether visual or auditory, increased the starting-delay of all age groups. There was no significant difference between off-road-visual and off-road-auditory distractors for any age group except for Grade 6 children. Off-roadauditory distractors increased starting-delay more than off-road-visual distractors for Grade 6 children. Furthermore, off-road and on-road distractors increased the number of missed opportunities for Grade 2 children. On-road distractors increased the number of missed opportunities of Grade 4 and Grade 6 children. The increase was greater for Grade 6 children.

Off-road-auditory (off-screen) distractors did not differently increase the starting-delay in comparison with off-road-visual distractors, which does not support the hypothesis. The difference was evident only for 10 - 11 year-old children, for whom off-road-auditory distractors increased starting-delay more than off-road-visual distractors. Nevertheless, offroad distractors, whether they were spatially distant from the main task or close to the main task, increased the starting-delay of all age groups, except for children aged 10 - 11 years. The study suggests that when distractors were off the road they had a distinctive effect of prolonging starting-delay. This effect was greater for 6 - 9 year-old children. However, whether these off road distractors were presented with the main task (off-road-visual distractors) or outside the main task (offroad-auditory distractors) did not have a significantly different effect, except for $10-11$ year-old children.
The effect of distractors was less evident for 10 - 11 year-old than for 6 - 9 year-olds, though distractors affected adults' starting-delay as much as it did for younger children. The current study suggests that young children were vulnerable to distractions off the road. Since no interaction was found between age and conditions with off-screen-auditory distractors, and since starting-delay was prolonged under the presence of off-screen-auditory distractors, this may suggest that all individuals in all age groups were distracted by off-screen auditory distractors. The effect of this type of distractor on decision-making skills suggests that adults were as vulnerable as children. This difference may support Jones's (1999) argument that the effects of auditory distractors on adults' performance depend on the type of main task.

The difference between the effect of off-road visual and off-road auditory distractors on missed opportunities could not be assessed statistically because of the low number of missed opportunities that occurred. However, by looking at the mean number of missed opportunities, it can be seen that for children aged $6-7$ years and $8-9$ years, there was a tendency to have a higher number of missed opportunities in the off-road visual condition. Such a tendency was not observable for $10-11$ year- olds1. However, overall, the study suggests that there was a higher number of missed opportunities in conditions with the presence of distractors. In addition, the results of this study suggests that $6-7$ year-old children had a higher number of missed opportunities in both conditions, with on-road and off-road distractors. The number of missed opportunities of 8 - 9 year-old and 10 - 11 yearold children increased in conditions with onroad distractors; the increase was greater for 10 - 11 year-old children. Adults did not have any missed opportunities. Although it was hypothesised that the number of missed opportunities would increase more in conditions with off-road distractors, since offroad distractors were stronger. This prediction was not supported. 
The high number of unsafe crossings in conditions without distractors was hypothesised to be the consequence of a low state of arousal. It was decided to conduct further analysis of the data. If unsafe crossings were a consequence of a low state of arousal, then they should have occurred more in the late trials than the early trials. The result shows that unsafe crossings happened to a similar extent in the early and the late trials. An examination of trials showed that a greater number of unsafe crossings happened in trials with two lanes than in trials with one lane. It also happened when trying to cross the road before the first car. This might suggest that young children do not try to look for traffic, because most unsafe crossings occurred at the start of the trial, before the first car. These attempts seem to be similar to the behaviour observed among young children, dashing into the street without looking (Zeedyk, et al. 2002). The presence of perceptual distractors had the advantage of catching the attention of individuals toward the road, making them more aware of the road situation so that the deficiency of not looking became less problematic in these conditions. If the distractors were in the direction of the cars, such as in the condition with on-road distractors (multiple cars), then the deficiency of not looking was overcome by the distractors.

As can be seen from the data the number of unsafe crossings was very low in conditions with on-road distractors. If, however, the distractors were not in the direction of the cars, they would not be beneficial anymore; rather, their presence would create a new problem of distractibility, especially for younger children.

The effect of different types of distractors on performance seems to depend on the type of variable assessed and age. Within task distractors also increased the number of missed opportunities, but this increase was accompanied with a reduction in the number of unsafe crossings. For Grade 2 children, both off- and within-task distractors increased the number of missed opportunities, though within task distractors enhanced performance by reduction in the number of unsafe crossings.

Suggestions for further research include assessing age differences in children's looking behaviour. In the current study it was suggested that the distractibility of young children by irrelevant information off the road might be related to their looking behaviour. However, it is not clear whether they do not know where to look or whether they know where to look but their sensitivity to distractions prevents them from looking at relevant information. Also, further research could investigate different types of distractors, especially those most common in traffic environments, such as sirens, car horns, etc.

A limitation of this study was the use of a computer-based pedestrian task. This simulation allowed us to control variables of interest and explore the factors that affect children's skills relevant to road safety, while protecting our child participants from the dangers of testing in real traffic environments. Also, previous researchers have supported the use of desktop simulations for pedestrian research (e.g., Seward, Ashmead \& Bodenheimer, 2006. However, it is acknowledged that the tasks had only face validity to the real pedestrian task. They might have under-represented the difficulties that children encounter when negotiating real traffic environments.

The findings of the current study could have implications for society as a whole and road safety educators in particular. Many people including drivers, police officers and teachers believe that children are responsible for the majority of accidents (Rivara, Bergman and Drake, 1989; Thomson,1991). The current study showed how children's skill is vulnerable to the complexity of the situation.

Therefore, to reduce the rate of pedestrian accidents, the behaviour of the other agent in the accidents, drivers, should be targeted as well. 


\section{REFERENCES}

Anderson, D. A., R. S. Huebner, Reed, J. R., Warner, J. C. and Henry, J. J. (1998), Improved Surface Drainage of Pavements: Final Report. NCHRP Web Document 16, Pennsylvania Transportation Institute, Pennsylvania State University.

Barbosa, R. S. (2011), Vehicle Dynamic Safety in Measured Rough Pavement, J. Transp. Eng., Vol.137, pp. 305-310.

Cenek, P, Davies, R, and Jamieson, N. (2012), Quantification of safety benefits resulting from road smoothing, Australasian Road Safety Research Policing Education Conference, Wellington, New Zealand.

ISO. (1995), Mechanical vibration road surface profiles-Reporting of measured data. ISO-8608, Geneva, 30.

Ong, G. P. and Fwa, T. F. (2007), Wet-Pavement Hydroplaning Risk and Skid Resistance: Modeling. ASCE Journal of Transportation Engineering, Vol. 133, No. 10, pp. 590-598.

Pacejka, H. B and Besselink, Igo (2012), Tire and vehicle dynamics, Oxford, UK.

Purdue Research Foundation (1974). Relating pavement roughness to vehicle behavior: final report, Federal Highway Administration.

Rose, J. G. and Gallaway, B. M. (1977), Water Depth Influence on Pavement Friction. ASCE Journal of Transportation Engineering, Vol. 103, No. 4, pp. 491-506.

Shi, X. M. and Cai, C. S. (2009), Simulation of Dynamic Effects of Vehicles on Pavement Using a 3D Interaction Model, J. Transp. Eng., Vol. 135, pp. 736-744.

Sun, L. (2002), Optimum design of "road-friendly" vehicle suspension systems subjected to rough pavement surfaces, Applied Mathematical Modelling, Vol. 26, pp. 635- 652

Sun, L. and Deng, X. J. (1998), Predicting Vertical Dynamic Loads Caused By Vehicle-Pavement Interaction, J. Transp. Eng., Vol. 124, pp. 470-478.

Wang, T. L., Shahawy, M. and Huang, D. Z. (1993), Dynamic Response of Highway Trucks Due To Road Surface Roughness, Computers \& Structures Vol. 49, No. 6, pp. 1055-1067. 